\title{
Long-Lasting Memories of Obstacles Guide Leg Movements in the Walking Cat
}

\author{
D. A. McVea and K. G. Pearson \\ Department of Physiology, University of Alberta, Edmonton, Alberta, Canada T6G 2H7
}

We examined the ways in which memories of previously seen obstacles can alter the stepping of walking cats. Cats were paused after the forelegs, but not the hindlegs, had stepped over an obstacle. Near the beginning of a variable delay period, the obstacle was lowered. On the subsequent step, the path of the hindlegs allowed us to make inferences about whether the memory of the obstacle was influencing leg movements. We present two main findings. First, the memory of the obstacle persisted for the duration that the animal straddled the original location of the obstacle. In one instance, this interval was $10 \mathrm{~min}$. Second, this memory includes information regarding the size and position of the obstacle relative to the animal. This information is used to plan foot placement and to redirect the step in mid-swing to avoid the previous position of the obstacle.

Key words: spatial memory; working memory; motor control; locomotion; vision; walking

\section{Introduction}

One essential task of the locomotor system is to avoid obstacles that impede walking. Important insights into the use of visual information for this task have come from the study of gaze direction in humans stepping over objects (Patla and Vickers, 1997) and with prescribed foot placement (Patla and Vickers, 2003). More detailed information about the neural structures responsible for this important behavior have come from studying walking in the cat, because it is possible to record simultaneously from supra-spinal regions and multiple muscles while the legs pass over a variety of obstacles (for review, see Armstrong and MarpleHorvat, 1996; Drew et al., 2002). One common finding from research in both humans and cats is that visual information about obstacles is not always used for on-line guidance or adjustment of leg movements. For example, humans walking toward an obstacle can accurately avoid it if they see it five steps before reaching its location (Mohagheghi et al., 2004), and cats walking through a cluttered walkway can avoid obstacles for three to four steps in the absence of vision (Wilkinson and Sherk, 2005). That on-line visual guidance is not always used is particularly obvious in the control of the hindlegs of the cat, which encounter an obstacle after the head and eyes have passed beyond its position (Drew et al., 1996).

The aim of the current study was to explore some aspects of the obstacle memory that guides the movements of the hindlegs of the cat over obstacles (because we are studying a previously undescribed aspect of a normal behavior, we use the simple term

\footnotetext{
Received 0ct. 18, 2005; revised Nov. 30, 2005; accepted Dec. 2, 2005.

This work was supported by the Alberta Heritage Foundation for Medical Research, the Canadian Institutes of Health Research, and the Natural Sciences and Engineering Research Council. We thank Drs. Atsumichi Tachibana, J. Maxwell Donelan, and Turgay Akay for their help collecting data and for their advice.

Correspondence should be addressed to Dr. D. A. McVea, Department of Physiology, University of Alberta, Edmonton, Alberta, Canada T6G 2H7. E-mail: dmcvea@ualberta.ca.

DOI:10.1523/JNEUROSCI.4458-05.2006

Copyright $\odot 2006$ Society for Neuroscience $\quad$ 0270-6474/06/251175-04\$15.00/0
}

"obstacle memory" throughout this study to refer to some persistent internal representation of an obstacle encountered during walking). Our first objective was to determine whether this obstacle memory could persist for longer than the time periods described in previous studies on obstacle avoidance during locomotion. To test this, we had cats walk partway over an obstacle and stop, with only the forelimbs having crossed the obstacle. The obstacle was subsequently dropped away while the animals were distracted, and we examined the kinematics of the hindlimb when the animal resumed walking. We next asked whether this obstacle memory can guide hindleg movements over multiple obstacles. This portion of the study allowed us to investigate the detail with which the memory of obstacles encountered during locomotion is encoded. To examine this issue, we observed cats while they walked partially over two obstacles before pausing to eat. One obstacle was immediately lowered, and the subsequent kinematics when the animal resumed walking allowed us to make inferences about the precision of the memory guiding the hindlegs.

The main findings of this study are that memories of obstacles encountered during locomotion are very persistent, lasting as long as the animal remained standing over the original location of the obstacle. Furthermore, these memories are encoded in a way that represents precise spatial information about the obstacle location.

\section{Materials and Methods}

In this study, we used two protocols to study the obstacle memory of walking cats. All procedures were done with two cats and were approved by the Health Sciences Animal Welfare Committee at the University of Alberta.

Protocol 1: one obstacle. In this protocol, cats were trained to walk partway over an obstacle $3.2 \mathrm{~cm}$ wide (the height of the obstacle was varied between 3 and $7.2 \mathrm{~cm}$ ). Animals were stopped from walking forward after their front legs had crossed the obstacle by presenting them with a food dish with sufficient food for them to eat for several minutes. 
As soon as the animal began eating in this position, the block was lowered so that the top became flush with the walkway surface. Between $5 \mathrm{~s}$ and 10 min after the animals straddled the obstacle, the animals were encouraged to walk forward so the hindlegs crossed where the obstacle had been.

Protocol 2: two obstacles. In this protocol, another obstacle, which measured $3.5 \mathrm{~cm}$ wide by $6.5 \mathrm{~cm}$ high, was placed in front of the same movable obstacle described above and was not lowered. The distance between the first (fixed) and second (lowered) obstacles varied from trial to trial, but the first obstacle was consistently placed so that a normal, unaltered step over it would intersect the position of the second obstacle. Cats were walked forward and presented with food so that both front feet crossed both obstacles, and one or both hind feet remained behind both obstacles. As in the one-obstacle protocol, the movable obstacle was dropped away and the animals were encouraged to walk forward after a delay period, which varied from trial to trial.

Training. Although cats were familiarized with the laboratory and the equipment described above, they were not trained to stand during the delay period. This allowed us to study a memory that was a natural part of locomotion but had the disadvantage that we could not continue trials beyond the duration for which animals would naturally stand. Many trials were unsuccessful because animals spontaneously sat down or walked backward.

Data collection and analysis. Reflective markers (diameter, $\sim 0.5 \mathrm{~cm}$ ) were placed on the toe of the right leg and the inner toe of the left leg. These markers were used to determine the kinematics of foot movements using the Peak Motus 8.2 motion analysis system (ViconPeak, Centennial, CO). Data were collected over a period of 3-4 weeks for both animals. Trials were conducted every $1-2 \mathrm{~d}$ for between 30 and $45 \mathrm{~min}$. The entire testing session was recorded to videotape and was later digitized off-line using the Peak Motus system. Trials in which the cat sat down, moved backward, or looked away from its food dish for an extended period of time were discarded.

\section{Results}

\section{Memories are persistent}

When cats step over an obstacle while walking, they produce a consistent pattern of hyperflexion of the forelegs and hindlegs (Drew et al., 2002). We first tested whether the increased flexion in the hindlegs would be present if the animal had the opportunity to see the obstacle but was paused for a delay period after the forelegs, but not the hindlegs, had stepped over the obstacle. In other words, we asked whether the obstacle memory that guides the hindlegs of cats over obstacles during normal walking could persist during long delays. Figure 1 shows the maximum step height after a delay period over an obstacle between 5.6 and 7.2 $\mathrm{cm}$ tall (there was no influence of obstacle height on maximum step height for this narrow range). These data show that cats will step significantly higher than normal even if paused for $>3$ min in one animal, and up to $10 \mathrm{~min}$ in another, before stepping over the obstacle. For the cat represented by the dots in Figure 1, control step height was taken from trials in which the cat paused in the position as in the experimental trials, but without the obstacle. For the cat represented by the open circles in Figure 2, control step height was taken from normal walking across the walkway. Data from the trailing steps were similarly higher than control steps (data not shown). The trials in Figure 1 represent all of the trials in which the animals remained standing and did not sit or turn around.

\section{Memories reflect detail of the object and its position}

We next examined the precision of the obstacle memory guiding the increased flexion in the step over the location of the obstacle. First, using obstacles of different heights, we found that step height after a delay period depended on the height of the obstacle. After a delay period over an obstacle $7.2 \mathrm{~cm}$ tall, the mean maximum height of the subsequent step was $9.8 \mathrm{~cm}(n=12)$. After a
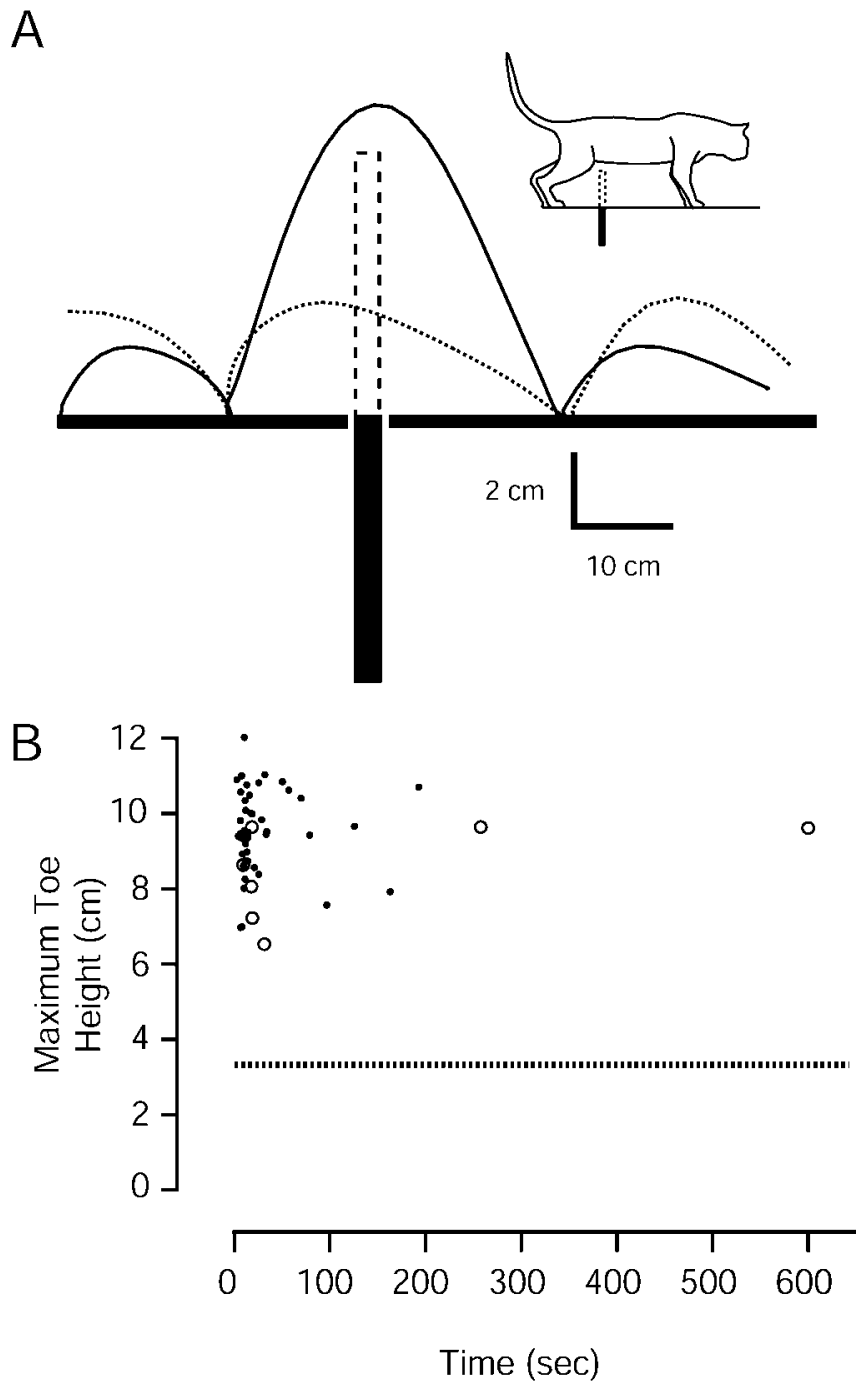

Figure 1. A, Toe trajectory during a leading step over a remembered obstacle (solid line). The delay period was $20 \mathrm{~s}$. The diagram in the top right corner shows the experimental set up. Cats stepped over an obstacle with the forelegs. The obstacle was lowered while the animal stood still eating. The dotted line shows the trajectory of a normal step without the obstacle for comparison. The dashed outline shows the original position of the obstacle. B, Maximum toe height during the leading step after a delay over a remembered obstacle (height varied between 5.6 and $7.2 \mathrm{~cm}$ ) for a variety of delays. The open circles and dots represent different animals. The dashed line is the mean toe height $(n=8)$ during control steps for the cat represented by the dots. The mean of control steps $(n=9)$ for the cat represented by the open circles was $3.1 \mathrm{~cm}$ (data not shown).

delay period over an obstacle $3.5 \mathrm{~cm}$ tall, the mean maximum height of the subsequent step was $6.7 \mathrm{~cm}(n=12)$. An unpaired $t$ test revealed these values to be significantly different $(p=3.4 \times$ $\left.10^{-6}\right)$. Second, Figure 2 shows that the trajectory of the cat's toe toward the obstacle varied depending on the distance between the toe and the obstacle at the time the step was initiated. Third, in trials involving two blocks (one of which was dropped away at the beginning of the delay), the paw generally landed between the two obstacles and subsequently passed above where the second obstacle had been, or passed above both obstacles in a single step (Fig. $3 A$ ). Occasionally, we observed a striking example of the detail with which foot movements were guided. Figure $3 B$ shows two examples. In the example in the top diagram, the foot was stopped and redirected in mid-swing to avoid crossing the previous position of the obstacle. This step followed a delay period of 


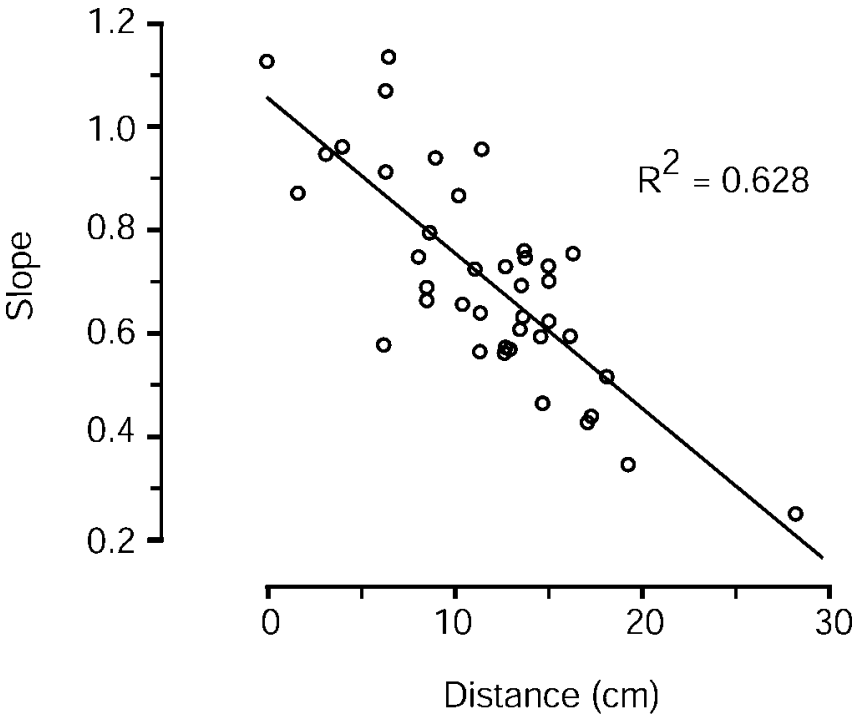

Figure 2. Leading steps of the hindlimbs after a delay over a remembered obstacle depend on the position of the toe. The slope from the toe position at the start of the step to the toe position at the maximum height varies inversely with distance from the remembered obstacle at the time of step initiation. The linear regression line and coefficient of determination are also shown.

$4 \mathrm{~s}$. In the bottom panel, the foot was lifted vertically until it was above the height of the remembered obstacle before being moved forward and over the previous position of the obstacle. This step followed a delay period of $10 \mathrm{~s}$.

\section{Discussion}

Although walking animals rely on visual input to avoid obstacles, vision is not always used for on-line guidance of leg movements. Both humans and cats can step accurately over obstacles they have not seen for a few seconds during normal walking (Patla and Vickers, 1997; Mohagheghi et al., 2004; Wilkinson and Sherk, 2005). In this study, we examined the duration and precision of one form of this memory, which, in cats, guides the hindlegs over an obstacle once the forelegs and the head have passed over it. Our strategy was to examine the leg movements of cats that had been paused for a delay period after the forelegs, but not the hindlegs, had stepped over an obstacle.

Our initial question was whether the memory of an obstacle encountered during walking would persist during an abnormal delay period, to allow subsequent steps to avoid the obstacle. The hindlegs of normal walking cats encounter obstacles up to $800 \mathrm{~ms}$ after the forelegs during walking (Drew et al., 1996). As shown in Figure 1, increased flexion was evident during all delays we examined. In most cases, this was between 10 and $200 \mathrm{~s}$, because this was the length of time animals usually remained standing. In a few cases, however, the delay exceeded $200 \mathrm{~s}$, lasting $600 \mathrm{~s}$ in one case. Large flexions were evident even after these exceptional delays. We were not able to determine the upper limit of the duration of this memory. Our attempts to test delays longer than a few minutes were usually prevented by the difficulty of keeping a cat standing and eating in one position for long periods of time. Nevertheless, we anticipate the memory of the obstacles may last considerably longer than $10 \mathrm{~min}$, because the one trial we obtained with a 10 min delay showed no decline in magnitude compared with the trials at shorter delays. Furthermore, we found no sign of a progressive decline over a period of $3 \mathrm{~min}$ in either animal, indicating this form of memory is very stable. We can be
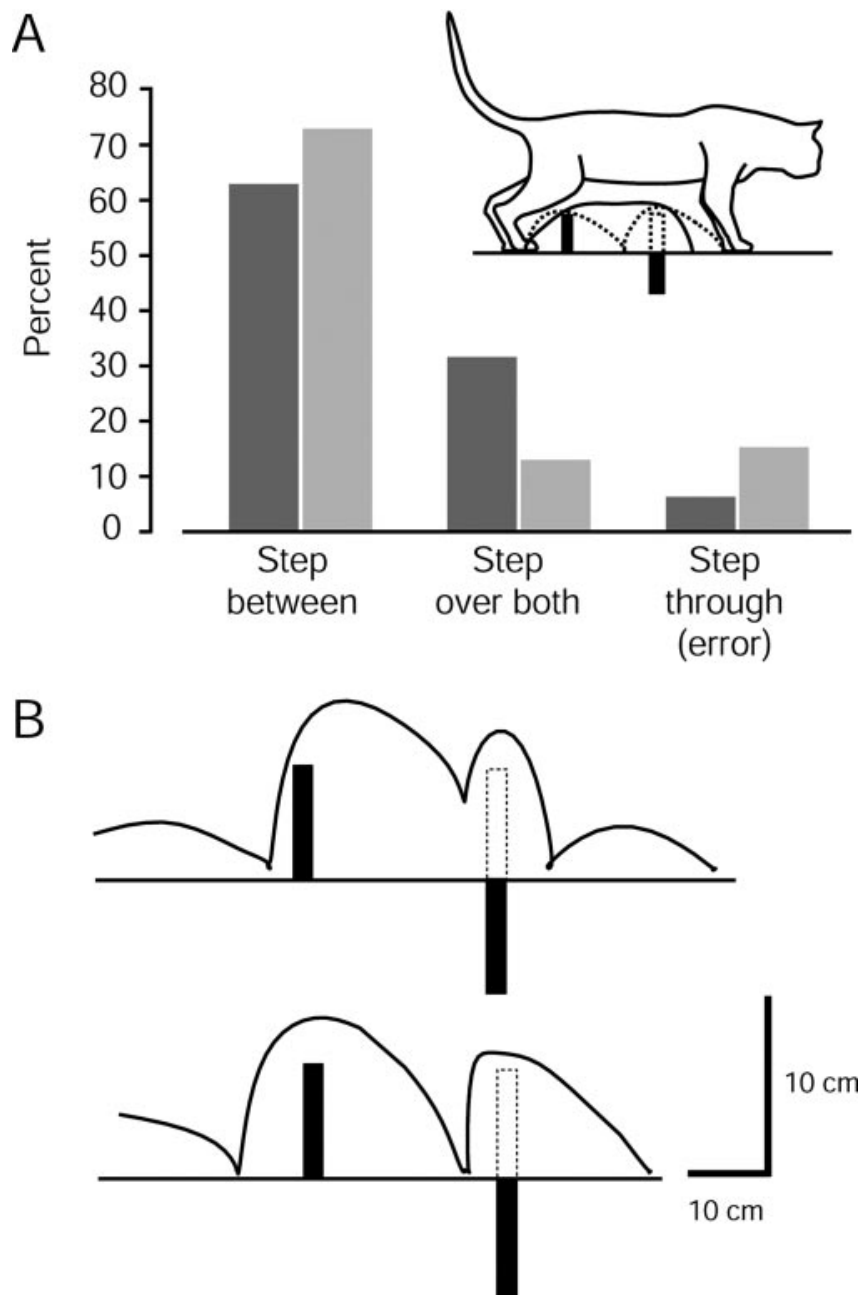

Figure 3. During stepping after a delay over two obstacles, cats demonstrate memory of obstacle location. $\boldsymbol{A}$, Steps after a delay over two remembered obstacles show memory of both obstacles. The protocol is shown in the inset. The animal was stopped and fed after the forelegs had crossed both obstacles and the hindlegs had crossed one or neither obstacle. Immediately afterward, the right obstacle was lowered, and the animal's forward progression was delayed by feeding. During the step after the delay, the animal could step between (dotted line), step over both (solid line), or step through the former position of the lowered obstacle (data not shown). The histograms show the percentage of steps (both leading and trailing) that were classified into each of these categories. The different shades represent two cats: dark gray, 78 steps; light gray, 40 steps. $\boldsymbol{B}$, Toe trajectories over two blocks showing precise memory of lowered block location. Top, A leading step in which the animal reversed direction in mid-swing, very close to the original position of the lowered block. Bottom, A trailing step in which the animal lifted the toe nearly vertically until it was just above the former position of the obstacle, before moving it forward.

confident the altered step was driven by memory and not by vision or somatosensory feedback, because the obstacle was absent during the delay period.

These long delays are at least one order of magnitude longer than those typically used in working memory tasks (Pasternak and Greenlee, 2005). For example, a recent pointing task to a remembered target noted decreases in accuracy after a $10 \mathrm{~s}$ delay (Lemay and Proteau, 2001), whereas a grasping study examined the effects of a 5 s delay on accuracy (Hu and Goodale, 2000). A recent study involving the cats' working memory of hidden objects found accuracy declined rapidly between delays of 0 and $30 \mathrm{~s}$ (Fiset and Dore, 2005). The memory we describe also lasts much longer than those described in other locomotion tasks that rely on memory. For example, cats can step accurately through a clut- 
tered walkway for between 2 and $3 \mathrm{~s}$ when relying on memory (Wilkinson and Sherk, 2005).

One possible explanation for the much longer-lasting memory of this study is that, during these long delays, animals held a simple memory that their next step would have to be high. This would be simpler and presumably more robust than a memory that carried detail about the obstacle and its position, and that guided the animals' movements once the step began. Four observations suggest the former is not the case. First, the height of the high step after the delay period varied depending on the height of the obstacle (Fig. $2 A$ ). Second, Figure $2 B$ shows that the trajectory of the cats' toe toward the obstacle varied depending on the distance between the toe and the obstacle at the time the step was initiated. This suggests that, during the delay period, the cats were aware of the position of the obstacle relative to their feet. The third observation that supports the contention that the high steps were guided by a detailed spatial memory of the obstacle came from trials involving two obstacles. In these trials, the large majority of steps took one of two trajectories, either landing between the two obstacles or passing above both. In the former case, the cat would have to make two altered steps to avoid the obstacles, first dropping the foot sharply after crossing above the first obstacle and then stepping higher than normal on the subsequent step to avoid the position of the second obstacle (which had been lowered). Only rarely did the toe pass through the position where the obstacle had been before it dropped away (Fig. 3). Because the cats we examined stepped either over both obstacles, or between them in such as way that they did not pass through where the obstacle had been, a simple memory of the obstacle that did not include information about its size and position would not be sufficient. Finally, the obstacle memory present during the delay period could be used to perform online corrections of stepping movements, as shown in Figure $3 B$. Note that these corrections were matched closely to the previous location of the obstacle. These four lines of evidence reflect a memory involving a precise representation of the obstacle relative to the body.

Given that the memory examined in this study includes information about the size and position of the obstacle relative to the body, it is worth considering why this memory lasts for significantly longer than either spatial memories examined in the context of reaching or saccades, or other forms of memories of obstacles during locomotion. One potentially relevant aspect of the memory we describe here is that the animal has both visual input about the size and shape of the obstacle and proprioceptive input related to the stepping of the forelegs. It may be that the action of the forelegs stepping over the obstacle provides essential information for establishing this memory. This combination of visual and proprioceptive input may be an important difference between this and other shorter-lived memories that guide movements during locomotion. Another relevant fact is that the memory task we used involves a natural, familiar task (stepping over an obstacle) interrupted midway. Furthermore, this memory task did not require the animals' attention. Unlike working memory tasks, most of which require the animals' attention (for an example, see Fiset and Dore, 2005; for review, see Pasternak and Greenlee, 2005; Goodale et al., 2004), the cats we studied were focused on their food as they ate, and not on the task. Importantly, they were not trained to stand or avoid the obstacle. One intriguing possibility, then, is that we are describing an essential feature of locomotion in cats that is short-lived during normal walking but can be extended for long periods of time. Cortical recordings from cats walking over obstacles provide some clues as to what areas of the nervous system might be involved in this behavior. Widajewicz et al. (1994) described cells in the motor cortex that were active for the interval after the forelimbs but before the hindlimbs had passed over an obstacle during normal, uninterrupted walking. It remains to be seen whether similar activity is evident in the motor cortex and other brain regions throughout the delayed stepping we describe in this study. If cortical regions are found in which neuronal activity is related to obstacle memory, then memory-guided stepping may provide an excellent paradigm for investigating a simple and robust form of working memory.

\section{References}

Armstrong DM, Marple-Horvat DE (1996) Role of the cerebellum and motor cortex in the regulation of visually controlled locomotion. Can J Physiol Pharmacol 74:443-455.

Drew T, Jiang W, Kably B, Lavoie S (1996) Role of the motor cortex in the control of visually triggered gait modifications. Can J Physiol Pharmacol 74:426-442.

Drew T, Jiang W, Widajewicz W (2002) Contributions of the motor cortex to the control of the hindlimbs during locomotion in the cat. Brain Res Brain Res Rev 40:178-191.

Fiset S, Dore FY (2005) Duration of cats' (Felis catus) working memory for disappearing objects. Anim Cogn 1-9.

Goodale MA, Westwood DA, Milner AD (2004) Two distinct modes of control for object-directed action. Prog Brain Res 144:131-144.

$\mathrm{Hu}$ Y, Goodale MA (2000) Grasping after a delay shifts size-scaling from absolute to relative metrics. J Cogn Neurosci 12:856-868.

Lemay M, Proteau L (2001) A distance effect in a manual aiming task to remembered targets: a test of three hypotheses. Exp Brain Res 140:357-368.

Mohagheghi AA, Moraes R, Patla AE (2004) The effects of distant and online visual information on the control of approach phase and step over an obstacle during locomotion. Exp Brain Res 155:459-468.

Pasternak T, Greenlee MW (2005) Working memory in primate sensory systems. Nat Rev Neurosci 6:97-107.

Patla AE, Vickers JN (1997) Where and when do we look as we approach and step over an obstacle in the travel path? NeuroReport 8:3661-3665.

Patla AE, Vickers JN (2003) How far ahead do we look when required to step on specific locations in the travel path during locomotion? Exp Brain Res 148:133-138.

Widajewicz W, Kably B, Drew T (1994) Motor cortical activity during voluntary gait modifications in the cat. II. Cells related to the hindlimbs. J Neurophysiol 72:2070-2089.

Wilkinson EJ, Sherk HA (2005) The use of visual information for planning accurate steps in a cluttered environment. Behav Brain Res 164:270-274. 